\title{
Criminologie
}

\section{Surveiller et punir (1976)}

\section{Jean-Paul Brodeur et Stéphane Leman-Langlois}

Volume 44, numéro 1, printemps 2011

Jean-Paul Brodeur, d'hier à aujourd'hui

URI : https://id.erudit.org/iderudit/1001601ar

DOI : https://doi.org/10.7202/1001601ar

Aller au sommaire du numéro

Éditeur(s)

Les Presses de l’Université de Montréal

ISSN

0316-0041 (imprimé)

1492-1367 (numérique)

Découvrir la revue

Citer cet article

Brodeur, J.-P. \& Leman-Langlois, S. (2011). Surveiller et punir (1976).

Criminologie, 44(1), 19-43. https://doi.org/10.7202/1001601ar

\section{Résumé de l'article}

Dans cet article, nous présenterons d'abord sous une forme schématique les principales thèses qui sont défendues dans le livre de Michel Foucault. Puis, nous consacrerons une seconde partie à examiner de façon plus approfondie les principaux points de l'argumentation qu'il développe et nous verrons comment certains des thèmes du livre nous paraissent avoir un intérêt plus immédiat pour le criminologue, soit parce qu'ils convergent avec la recherche en criminologie ou parce qu'ils en constituent une critique. Ensuite, nous soulèverons certains points qui concernent la méthode utilisée par Foucault. Cette partie de notre exposé sera plus critique. Enfin, nous conclurons par un rappel des conclusions de Foucault sur la naissance de la criminologie. Nous tenterons alors de mettre en lumière ce que les études criminologiques devraient retenir du livre de Michel Foucault, outre ce qu'il nous apprend à titre documentaire sur la naissance de la prison.
Ce document est protégé par la loi sur le droit d'auteur. L'utilisation des services d'Érudit (y compris la reproduction) est assujettie à sa politique d'utilisation que vous pouvez consulter en ligne.

https://apropos.erudit.org/fr/usagers/politique-dutilisation/ 


\section{Surveiller et punir}

Jean-Paul Brodeur $\left(1976^{\star}\right)$

\section{Note introductive}

En 1975 paraît Surveiller et punir, de Michel Foucault. L'ouvrage aura l'effet d'un pavé dans la mare de la criminologie, pour plusieurs raisons, mais pour deux en particulier. La première est qu'un intellectuel internationalement respecté y fait une histoire de la prison qui révèle le fonctionnement sociopolitique d'une institution dont on ne voulait jusqu'alors savoir que deux choses: fait-elle diminuer le crime? et, estelle «humaine»? Or, Foucault démontre que le fait de poser ces questions, qui n'ont que l'apparence d'une critique de la prison, vient au contraire renforcer les fondements conceptuels de son existence, qui ne sont jamais remis en question: les crimes existent, on ne peut pas les réparer, il faut donc agir sur leurs auteurs. La seconde raison qui fit de Surveiller et punir un must read pour les criminologues est la mise en cause radicale de leurs savoirs et de leurs pratiques, leur discipline, qui sert à réifier une série de choix politiques ayant pour but de renforcer le déséquilibre de pouvoir entre dominants et dominés. Ses sujets principaux, prisonniers, délinquants, criminels, victimes, prisons, policiers, etc., elle les prend tout bonnement comme des réalités naturelles en ignorant les processus, interactions et structures qui les produisent. Dans sa revue du livre, Jean-Paul Brodeur relève, compare et critique avec brio les principales thèses de Foucault. Il passe en revue les trouvailles, les oublis et les principales failles méthodologiques et logiques du philosophe, apportant un éclairage hors pair sur l'ouvrage. Un ouvrage qui, somme toute, reste d'actualité: la criminologie n'a pas changé, la

${ }^{\star}$ Criminologie, XIX (1-2), 196-218. 
prison non plus. L'analyse qu'en fait Brodeur, elle aussi, aurait pu être écrite la semaine dernière. Par exemple, un passage sur la collusion entre syndicats, crime organisé, fonctionnaires et politiciens est tellement d'actualité qu'on s'en arrache les cheveux. Et on peut parier que, comme en 1976, peu de criminologues s'y intéresseront, contrairement aux thèmes à la mode comme les prédateurs sexuels, les pédophiles ou même les «cybercriminels».

Stéphane Leman-Langlois ${ }^{\star \star}$

RÉSUMÉ - Dans cet article, nous présenterons d'abord sous une forme schématique les principales thèses qui sont défendues dans le livre de Michel Foucault. Puis, nous consacrerons une seconde partie à examiner de façon plus approfondie les principaux points de l'argumentation qu'il développe et nous verrons comment certains des thèmes du livre nous paraissent avoir un intérêt plus immédiat pour le criminologue, soit parce qu'ils convergent avec la recherche en criminologie ou parce qu'ils en constituent une critique. Ensuite, nous soulèverons certains points qui concernent la méthode utilisée par Foucault. Cette partie de notre exposé sera plus critique. Enfin, nous conclurons par un rappel des conclusions de Foucault sur la naissance de la criminologie. Nous tenterons alors de mettre en lumière ce que les études criminologiques devraient retenir du livre de Michel Foucault, outre ce qu'il nous apprend à titre documentaire sur la naissance de la prison.

MoTS-CLÉS - Michel Foucault, prison, système carcéral, pénalité, panoptisme, hard datas, soft datas.

\section{Introduction}

Michel Foucault vient de publier sous le titre de Surveiller et punir ${ }^{1}$ un livre qui comporte un intérêt considérable pour les études criminologiques. Il suffit, pour le faire percevoir, de remarquer que le sous-titre du livre de Foucault est Naissance de la prison. Nous allons tenter dans ce texte de rendre compte de Surveiller et punir, en mettant l'accent sur les points du livre qui sont susceptibles d'intéresser davantage les criminologues. Il est une autre lecture du livre, qui en soulève les enjeux philosophiques, sur laquelle nous insisterons moins, quitte à nous y référer sur un mode allusif, à quelques reprises. Notre compte rendu comprendra cinq parties: 1) nous présenterons d'abord sous une forme

\footnotetext{
${ }^{\star \star}$ Chaire de recherche du Canada en surveillance et construction sociale du risque École de service social, Université Laval stephane.leman-langlois@svs.ulaval.ca

1. Michel Foucault (1975), Surveiller et punir, Paris, Éditions Gallimard.
} 
schématique les principales thèses qui sont défendues dans le livre de Michel Foucault; 2) nous consacrerons une seconde partie à voir de façon plus approfondie les principaux points de l'argumentation qu'il développe; 3) nous allons ensuite brièvement commenter certains des thèmes du livre qui nous paraissent avoir un intérêt plus immédiat pour le criminologue, soit qu'ils convergent avec la recherche en criminologie ou qu'ils en constituent une façon de critique; 4) nous soulèverons, dans une quatrième partie, certains points qui concernent la méthode utilisée par Foucault. Cette partie de notre exposé sera plus critique que les autres; 5) nous conclurons par un rappel des conclusions de Foucault sur la naissance de la criminologie elle-même. Nous tenterons alors de mettre en lumière ce que les études criminologiques devraient retenir du livre de Michel Foucault, en outre de ce qu'il nous apprend à titre documentaire sur la naissance de la prison.

Nous rappellerons très rapidement, avant de nous engager plus avant dans notre exposé, que Surveiller et punir représente moins le résultat de nouvelles préoccupations de la part de Michel Foucault, qu'une tentative pour articuler de façon plus étroite la double thématique dont s'était nourri, jusque-là, son travail. L'intérêt le plus ancien de Michel Foucault réside dans l'histoire, conjointement menée, du type de regard qui est porté sur les individus par la psychiatrie naissante (Histoire de la folie à l'âge classique) et par la médecine (Naissance de la clinique). Les pratiques coercitives et les diverses institutions (asile, hôpital, prison) auxquelles ont donné lieu le regard de la médecine et celui de la psychiatrie ont fait de ce cadre l'objet d'une attention particulière de la part de Foucault. Dans deux livres subséquents - Les mots et les choses et L'archéologie du savoir - Michel Foucault a généralisé la problématique régionale développée dans les deux livres précédents; il a étendu à toutes les sciences humaines ses préoccupations à l'égard de l'histoire de la formation d'un savoir positif sur l'homme et il a tenté de décrire l'émergence de quelques-unes parmi les principales sciences de l'homme, depuis l'époque classique. Les deux motifs des recherches de Foucault - la genèse des objets à partir de la qualité d'un regard et la naissance des disciplines sous la pression des institutions et de leur mutation - sont couplés l'un à l'autre de façon préméditée dans Surveiller et punir. Les formes institutionnelles abordées de façon régionale dans l'Histoire de la folie et dans la Naissance de la clinique y sont totalisées sous la forme d'un ensemble plus vaste des divers systèmes de coercition et de discipline qui sera finalement désigné comme le carcéral et celui-ci est posé, par hypothèse, 
au fondement de la formation des savoirs - des sciences humaines - qui produiront l'homme comme individu (délinquant).

\section{Partie 1}

1. Le livre de Michel Foucault se présente, au premier abord, comme la réponse à deux questions qui sont explicitement énoncées dans le livre et comme la réalisation partielle d'un objectif dont la formulation donne moins lieu à l'énoncé d'une question explicite qu'à l'affleurement continue d'une interrogation qui traverse tout le livre. Les thèses qui sont impliquées en réponse aux deux questions soulevées et la prise de parti qui est présupposée par la poursuite de l'objectif que se donne le livre peuvent être mises en correspondance avec les quatre règles que Foucault affirme suivre dans son livre (p. 28). Nous énoncerons d'abord les thèses auxquelles nous venons de nous référer et nous effectuerons ensuite brièvement une mise en correspondance de ces thèses avec les règles qui sont posées par Foucault au début du livre.

Première thèse. On peut caractériser la pénalité sous l'ancien régime en France (sous la monarchie) de la façon suivante: il s'agit d'une pénalité de supplice. Les caractéristiques d'une telle pénalité sont les suivantes: 1) son objet est constitué par le corps du condamné, que le supplice, dans sa forme paroxistique, a pour objet de détruire; 2) son application, participant du caractère exceptionnel d'un cérémonial éclatant, n'est pas systématique; 3) par son excès même - l'anéantissement du corps supplicié - le supplice a pour fonction de manifester la puissance du souverain, défiée par le criminel. Un certain nombre de facteurs, sur lesquels nous reviendrons, amenèrent les législateurs de la Révolution française à procéder à une réforme complète de cette pénalité. Les principes mis de l'avant par les réformateurs étaient rigoureusement à l'inverse des précédents. 1) Le châtiment était investi d'une double fonction: il devait à la fois corriger le délinquant et dissuader le corps social de suivre son exemple; 2) d'où son point d'application: ne plus frapper le corps mais l'âme. D'une part, l'âme du délinquant, qui devait acquérir les habitudes de la vertu et, d'autre part, l'âme des divers membres du corps social, pour lesquels le châtiment du délinquant devait constituer un signe faisant obstacle aux volontés de délinquance (p. 96) ; 3) afin de remplir cette fonction, le châtiment devait posséder au moins deux caractères: il devait d'abord, pour acquérir la valeur d'un signe de dissuasion, avoir les prédicats d'un symbolisme 
transparent, c'est-à-dire être exactement mesuré par le type d'offense qu'il sanctionnait. Le meurtrier serait tué à son tour, l'empoisonneur serait aspergé de poison et ainsi de suite; le châtiment devait être parlant pour l'esprit. Second caractère: le principe de toute publicité, serait-ce une publicité de dissuasion, réside dans sa systématicité. Aucun espace ne doit lui échapper. L'application des peines devait donc être coextensive à tout le champ des illégalités.

Tels étaient les principes de la réforme. Or il s'est trouvé que ceux-ci ont été très rapidement pervertis par la réalité de la réforme pénale. En effet, l'emprisonnement ayant vite constitué le seul châtiment imposé, deux des principes précédemment posés se sont trouvés rejetés: d'une part, les châtiments cessent d'être individualisés et adaptés aux crimes qu'ils sanctionnent; surtout, il semble que l'on ait renoncé à faire de l'âme le point d'application des châtiments. Le système carcéral est en effet défini par Foucault comme une technologie politique des corps (p. 28). De cette perversion de la réforme pénale suit la question centrale du livre: comment expliquer qu'une critique d'une pénalité centrée sur le supplice ait produit une pénalité de l'enfermement des corps au lieu d'une économie des châtiments qui ait pour objet premier la contrainte des esprits? La réponse à cette question, qui occupe la majeure partie du livre de Foucault, constitue la première des thèses qui y est défendue. Cette thèse, énoncée de façon sommaire est la suivante: le pouvoir judiciaire, qui voulait à l'origine se réaliser sous la forme d'une tutelle des âmes, a été colonisé par une technologie des corps (p. 141). Cette technologie des corps, sous l'impulsion de diverses pressions des pouvoirs, s'était progressivement élaborée sous les traits de la discipline, dans des champs d'activités aussi diversifiés que ceux que représentent respectivement l'atelier de travail, l'hôpital, l'école et le camp d'armée. Ce sont ces habitudes disciplinaires acquises pendant les $\mathrm{XVIII}^{\mathrm{e}}$ et $\mathrm{XIX}^{\mathrm{e}}$ siècles qui ont très rapidement subverti la réforme pénale dont les principes avaient été formulés à la Révolution française et qui l'ont contrainte à se réaliser comme une technologie politique des corps, dont le modèle est donné par la colonie pénitentiaire. Nous nous référerons à cette première thèse sous le vocable de thèse historique, dans la suite de ce texte. Notons que le problème qui donne naissance à cette thèse est lui-même conditionné par l'affirmation de Foucault que l'incarcération est avant tout un mode original de rapport au corps. Si le carcéral était défini comme un rapport à l'âme plutôt qu'au corps, le problème historique que veut résoudre Foucault - la trahison de la réforme de la pénalité des supplices - ne se poserait pas. Il faut donc 
voir dans la proposition qui lie le système carcéral à une technologie des corps la pierre angulaire de tout le livre de Foucault.

Seconde thèse. Foucault établit de façon assez spectaculaire (p. 270 et suiv.), non seulement que la prison n'a pas été désirée par le système judiciaire mais que ses déficiences ont été très tôt reconnues. Il se plaît à mettre en parallèle deux critiques de la prison, dont l'une s'énonce un peu avant la seconde moitié du XIX ${ }^{e}$ siècle alors que l'autre est beaucoup plus rapprochée de nous (1945): ces critiques sont essentiellement les mêmes et elles se disent dans les mêmes termes. D'où une seconde question, plus tardive dans le livre: comment expliquer qu'une institution qui s'est imposée de l'extérieur au judiciaire et dont les défauts ont été immédiatement reconnus se soit maintenue jusqu'à nos jours et d'où vient que les initiatives pour l'abolir se heurtent encore à une résistance décidée? La réponse qu’apporte Foucault à cette double question est, il faut le dire, moins claire que celle qu'il apporte à la question précédente. Telle semble être la thèse qu'il nous propose: la prison constitue la pièce essentielle d'une gestion politique des illégalismes (p. 277). Son rôle est de substituer au fond sauvage des illégalismes une délinquance politiquement inoffensive, constituée d'individus facilement repérables puisqu'ils portent les stigmates d'un séjour dans une colonie pénitentiaire, et qui est d'autant plus contrôlée par le pouvoir politique qu'elle en est la création et bien souvent, l'instrument. Cette délinquance apprivoisée et marginalisée est en effet systématiquement exploitée par les pouvoirs en place, qui en tirent un nombre considérable de bénéfices. Nous fournirons plus loin une illustration québécoise de ce qui nous semble être la thèse de Foucault, que nous ne développerons pas davantage pour l'instant. Nous désignerons dorénavant cette seconde thèse par les termes de thèse sociologique ou socio-judiciaire.

Troisième thèse. Nous nous contenterons d'énoncer rapidement la troisième thèse de Foucault. Elle ne figure qu'à titre d'objectif et de règle qui demeure relativement abstraite et d'application allusive, dans un livre qui doit servir d'arrière-plan à des recherches futures (voir la note finale, p. 315). Cette thèse peut s'énoncer sous la forme d'une décision méthodologique d'articuler les unes sur les autres dans l'horizon d'effets de renforcement réciproque, les structures qui définissent le pouvoir et celles qui définissent le savoir. Le savoir détermine les modalités des stratégies utilisées par le pouvoir pour contraindre et le pouvoir constitue les nouveaux champs d'exercice du savoir en lui assignant ses objets. La vérité d'une archéologie du savoir réside dès 
lors dans une généalogie de la constitution d'un complexe politico-scientifique où le savoir légitime l'exercice d'un pouvoir qui lui fournit son aliment. Nous réserverons à cette thèse l'appellation de thèse épistémologique.

On peut maintenant établir un bref parallèle entre ces différentes thèses et les quatre règles que Foucault affirme avoir suivi dans son travail (p. 28). Les règles un-privilégier la perspective de la tactique politique dans l'étude des châtiments - et quatre - voir dans la nouvelle pénologie un mode transformé de rapport au corps - s'unissent pour produire la première thèse de Foucault, qui a précisément pour but d'expliquer comment une réforme pénale qui avait pour dessein de circonvenir les âmes s'est métamorphosée en un projet politique de discipliner les corps. La troisième règle - rattacher à une matrice commune le savoir criminologique et un type de système pénal - est une reformulation de l'objectif que nous avons énoncé sous la forme d'une troisième thèse. La seconde des règles que suit Michel Foucault - privilégier la recherche des effets positifs du système pénal sur la société sur la simple description de ses fonctions répressives (négatives) - donne naissance à la seconde des thèses dont nous avons rendu compte et qui s'interroge sur le rôle effectif que joue cette sphère de délinquance qui est l'un des produits du système pénal.

\section{Partie 2}

2. Nous allons maintenant tenter de retracer le mouvement général de l'argumentation développée par M. Foucault. Qu'il soit entendu que le poids de cette argumentation concerne davantage la première thèse ou thèse historique, dont la défense fait l'objet de la partie centrale du livre, que la seconde thèse, qui apparait sous une forme explicite seulement vers la fin du livre (on y fait souvent allusion ailleurs cependant). Le défaut d'une argumentation dont la base empirique soit suffisamment assurée constitue d'ailleurs l'une des faiblesses du livre pour ce qui est de la thèse socio-judiciaire qu'il nous propose. Bien que Foucault s'y réfère souvent, la thèse épistémologique ne fait pas l'objet d'une démonstration effective dans Surveiller et punir, qui nous renvoie à des recherches futures. Nous suppléerons pour notre part, ce qui nous semble être l'un des indices de sa justesse.

2.1 Soit donc trois types d'organisation des châtiments et leur produit respectif. Premier modèle: le supplice. Son produit est la marque que 
laisse sur le corps du criminel le corps du souverain, dont le vicaire est le bras du bourreau, lui-même officiant de rituels exécutoires terrifiants qui ont précisément pour objet de manifester cette puissance du prince. Second modèle: la cité punitive. Son principe est le quadrillage de tout l'espace social et son produit est le signe public qui doit représenter de façon continue à tous les esprits l'issue fatale des carrières criminelles, pour les dissuader de s'y engager. Dernier modèle: l'institution corrective ou pénitentiaire. Son produit est la trace indélébile que laissent sur les corps les dressages et les servitudes multiples dont il est l'objet dans la colonie pénitentiaire (pour cette triple distinction, voir la p. 134). Le problème historique est double: il faut d'abord expliquer pourquoi on a voulu substituer la contrainte des âmes au supplice des corps; il faut ensuite expliquer pourquoi voulant passer du premier au second modèle, on n'a en fait réussi qu'à instaurer le troisième.

La réponse à la première question tient dans la volonté des réformateurs d'adapter la pénalité à l'évolution de la criminalité et aux mutations sociales qui l'ont déterminée. Du Moyen-Âge à la révolution industrielle, l'Occident passe graduellement d'une criminalité de sang à une criminalité de fraude (p. 80). Le trait déterminant de la première est son caractère soudain et éruptif auquel correspond un exercice des châtiments qui est à la fois violent et irrégulier (événementiel). La seconde est par contraste diffuse et répandue dans l'ensemble du corps social. Pour conjurer ces illégalismes qui sont coextensifs à la totalité du corps social, il faut lui affronter un exercice des châtiments qui recouvre lui-même la société dans son entier et dont l'opération soit parfaitement régulière. Il est d'abord apparu que le moyen le plus économique d'instaurer le changement d'échelle considérable qui était impliqué par le passage d'une pénalité de l'exception à un quadrillage judiciaire de tout l'espace social, était de conférer au crime une existence sociale publique dont la forme serait celle du signe. Ce recours au vieux principe de l'exemplarité du châtiment ne doit pas s'interpréter comme la reprise non critique d'un expédient ancien. Il s'effectue plutôt sous les traits de la constitution d'une sémio-technique (technique des signes) qui obéit à des règles qui sont le produit d'un savoir ambitionnant la rigueur et que Michel Foucault décrit avec précision (p. 96-103). L'effet de dissuasion qui était auparavant naïvement obtenu par la sévérité des peines le sera désormais en maximisant au plan des représentations la rigueur de châtiments qui sont en réalité considérablement adoucis (dans le but premier de préserver du remords ceux qui s'autorisent à les 
appliquer) et en inculquant au corps social, pour équilibrer la désescalade des peines, la certitude absolue de l'avènement du châtiment pour toute infraction commise. C'est cette certitude qui confère à la représentation du crime la valeur d'un signe: tout crime doit être le signe d'un châtiment futur.

Venons-en maintenant à la seconde question: comment a-t-on substitué le dressage des corps au projet d'arraisonner les âmes? Nous avons déjà donné sous la forme de la thèse historique la réponse que $M$. Foucault apporte à cette question et qui tient dans une importation par le judiciaire de techniques d'ordre disciplinaire. Reste à voir comment Foucault défend cette thèse. La stratégie argumentative qu'il utilise est la suivante: en affirmant nous rendre compte des diverses caractéristiques des pratiques disciplinaires, Foucault va nous fournir une description qui de façon croissante va se mettre à coïncider avec ce que nous pressentons tous intuitivement être la réalité carcérale. Nous sommes dès lors prêts à accueillir la thèse historique qu'en instaurant les disciplines, le $\mathrm{XIX}^{\mathrm{e}}$ siècle a créé la prison. L'argumentation déployée par Foucault procède en trois étapes.

Dans les deux premiers chapitres de la partie de son livre intitulée Discipline, Michel Foucault va nous décrire les fonctions qu'ambitionnent de remplir les techniques disciplinaires, les effets qu'elles désirent obtenir, ainsi que les moyens qu'elles utilisent pour y parvenir. Les fonctions que remplit une technique disciplinaire sont au nombre de quatre et Foucault leur assigne à chacune leur symbole. 1) La discipline détermine d'abord la place qu'occupent les individus dans l'espace physique et social et elle les y fixe de façon définitive. Le symbole de cette opération est la constitution de ces tableaux synoptiques qui quadrillent exactement un champ épistémologique. 2) Elle soumet le déroulement des actes à une programmation rigoureuse dont la fin est de mettre le corps en symbiose avec les outils de son travail. Le prototype d'une telle unité symbiotique est la manœuvre du soldat qui fait totalement corps avec son arme. 3) Elle parcellarise l'activité humaine en la découvrant sous la forme de tâches graduées dont l'expression temporelle est constituée par une multiplicité de séquences orientées, indéfiniment répétables et dont le modèle est l'exercice militaire. 4) Les segments d'activité précédemment découpés composent ensemble un champ de forces obéissant à une géométrie précise dont l'un des exemples - cité par Foucault - est la tactique militaire. On pourrait aussi invoquer la division industrielle du travail. Discipline, on le voit, s'entend ici à la façon 
habituelle puisque trois des symboles de ses fonctions - la manœuvre, l'exercice et la tactique - sont empruntés à l'art militaire. Les moyens utilisés par la discipline pour obtenir ces effets sont, quant à eux, au nombre de trois: ce sont respectivement la surveillance, la sanction de tout comportement qui s'écarte d'une norme édictée et l'examen qui sert précisément à vérifier le degré de conformité des individus et de leurs comportements à des moyennes établies.

L'examen, écrit Foucault (p. 189), intervertit l'économie habituelle de la visibilité: au lieu de manifester un souverain à ses sujets, il révèle au contraire, sous un rapport qui a été prédéterminé, les individus qui y sont assujettis à un pouvoir qu'il contribue à dissimuler. Il faut voir dans cette inversion la clé de l'idéal qui habitera désormais le système pénal et que Foucault désigne par le terme panoptisme ${ }^{2}$. Au vrai, il n'est pas surprenant que la réforme pénale qui voulait produire le crime sous l'espèce du signe ait avorté; dans la mesure où elle achoppait à transformer un rapport de visibilité qui avait été défini par l'Antiquité grécolatine, elle demeurait encore essentiellement tournée vers le passé. Le visible se caractérisait, depuis l'Antiquité, comme une relation établie entre un nombre restreint d'objets - temples, spectacles et manifestations du pouvoir souverain - et la multitude des regards. Tel était aussi le principe d'une sémio-technique dont l'objectif était la dissuasion: une somme limitée de signes serait offerte à tous les regards. Le panoptisme, qui constitue l'idée régulatrice d'un état policier, intervertit les termes de cette relation: c'est le comportement de la totalité des membres du corps social qui est dorénavant observé par le petit nombre de ceux qui détiennent le pouvoir et leurs auxiliaires. Le système pénal cesse de vouloir exclusivement proposer des exemples à la multitude pour s'investir dans une généralisation des mécanismes de surveillance, qui jusque-là étaient appliqués dans des circonstances exceptionnelles (p. 211). Par cette volonté de généralisation, le troisième modèle du système pénal retient l'un des traits originaux du second modèle, qu'il supplante. Le terme de panoptisme est emprunté par Foucault à Jeremy Bentham, le père de l'utilitarisme, qui sous le terme de Panopticon esquisse les plans d'un type d'édifice dont la multiplication lui apparaît souhaitable pour rentabiliser davantage les différents types d'entreprises. Il s'agit, en gros, d'une espèce de ruche en pierre, en forme d'an-

2. Du grec «pan» qui signifie «tout» et de la racine verbale d'un verbe grec signifiant «voir». 
neau, dont les alvéoles sont des cellules et dont le centre vide est occupé par une tour de surveillance d'où le regard peut se porter sur toutes les cellules. Le fait qu'il n'y ait eu que les seules prisons qui aient été édifiées en conformité avec les instructions de Bentham ne contribue pas peu à accréditer la thèse de Foucault selon laquelle ce qui est décrit comme technique disciplinaire et qui culmine dans le projet d'ériger des maisons panoptiques a été partie prenante dans l'infléchissement du système pénal vers la multiplication des colonies pénitentiaires.

Cette thèse recevra d'ailleurs au moins un début de vérification dans la description qu'il fera des prisons dans la dernière partie de son livre. Il se révélera que tout ce par quoi la prison excède la simple maison de détention, qui existait sous une forme ou une autre depuis le début des âges, lui a été apporté par l'intégration de techniques disciplinaires isolement spatial des détenus, obligation de se livrer à des travaux programmés, visée corrective (p. 251 ; voir aussi p. 274 et 275).

2.2 La thèse socio-judiciaire caractérise la délinquance comme étant une colonie du pouvoir politique. Cette thèse donne plutôt lieu à des illustrations qu'à une démonstration en règle par $M$. Foucault. Avant de l'illustrer pour notre propre compte, nous tâcherons de mieux l'entendre. Foucault assigne une double généalogie à la délinquance. Le délinquant est une création épistémologique: il se distingue du simple infracteur par un transfert généralisant des propriétés apparemment déviantes de certains de ses actes à toute sa personnalité - sa vie (p. 255). Celle-ci, conçue sous l'espèce de l'anormalité, est caractérisée de façon étiologique comme un réseau d'instances causales - pulsions psychologiques, déterminismes biologiques ou sociologiques, etc. - qui peuvent être mises en corrélation avec des comportements pour ainsi produire l'illusion d'un savoir. La délinquance, que l'on se représentera cette fois comme le regroupement dans un espace marginal du corps social des individus réputés délinquants, est elle-même le produit du pouvoir judiciaire dans sa variante carcérale. Un délinquant se définit intuitivement et à toutes fins pratiques comme quelqu'un qui a eu des démêlés avec la justice. Je crois que la métaphore la plus propre à faire saisir la pensée de Foucault sur le sujet est celle du ghetto; la production par le système pénal d'un regroupement des délinquants est homologable à la constitution d'un ghetto dans la cité des illégalismes. La fonction et l'effet principal d'un ghetto, comme celle de tous les quartiers réservés, est de faciliter le contrôle et l'exploitation de ceux qui y 
sont entassés. Telle est aussi la fonction de la constitution d'une délinquance socialement identifiée. Fonction de contrôle d'abord: on a tort de penser que seuls les individus qui sont effectivement incarcérés sont sous le contrôle du pouvoir judiciaire. En réalité, l'existence des casiers judiciaires, des conditions de probation et des différentes fiches policières, qui détermine le regroupement des détenus à leur sortie de prison en un «milieu» marginalisé par les employeurs et infiltré par divers réseaux d'indicateurs, assure le caractère illusoire de leur libération. La prison s'étend bien au-delà de ses murs. Fonction d'exploitation, ensuite. Cette exploitation est double. Elle peut d'abord prendre une forme directe. Foucault cite en ce cas, sans le développer, l'exemple de la prostitution (p. 285). Cet exemple est assez bien choisi puisque l'on peut observer que la distribution des profits s'y effectue, d'une certaine façon, en raison inverse de la délinquance de ceux ou de celles qui s'y livrent. La personne la plus délinquante au regard de la loi, la prostituée elle-même, est bien souvent la personne qui en retire le moins de bénéfices et qui est soumise à la plus grande exploitation. La délinquance peut aussi être exploitée indirectement en constituant l'objet substitutif d'une répression qui répugne à affronter les illégalismes véritables qui affligent le corps social, soit qu'elle les craigne ou qu'elle s'en fasse complice.

La situation qui prévaut actuellement au Québec dans les milieux syndicaux de la construction offre un bon exemple des procédures de colonisation de la délinquance par les pouvoirs publics. Les deux formes de l'exploitation de la délinquance s'y manifestent de façon transparente. Exploitation directe d'abord: le rapport de la commission d'enquête établit sans l'ombre d'un doute que des officiers gouvernementaux qui occupaient de hautes fonctions dans l'entourage du premier ministre étaient en liaison avec les marges délinquantes du syndicalisme dans le but d'assurer la paix sur les chantiers. Exploitation indirecte, ensuite: composées d'individus qui pour une large part sont déjà repérés par la police et qui possèdent un casier judiciaire, les marges délinquantes du syndicalisme constituent par leur vulnérabilité même l'objet tout désigné d'une répression qui, menée à grand renfort de publicité, tentera de faire oublier l'illégalisme foncier des représentants du gouvernement dans cette affaire. Il est en outre symptomatique de remarquer que la délinquance syndicale fait l'objet de poursuites criminelles alors que la mainmise du gouvernement sur les terres des Indiens dans la région de la baie James, qui constitue un illégalisme d'une tout autre échelle que 
la délinquance syndicale, ne fait l'objet que d'une procédure civile. Il faut noter enfin que l'exploitation substitutive de la délinquance syndicale comporte une plus-value politique directe: la mise au pas, par le moyen de tutelles, de syndicats dont le militantisme politique était devenu inquiétant pour le pouvoir politique.

2.3 Nous reviendrons dans la prochaine section sur la thèse que nous avons qualifiée d'épistémologique et dont l'élaboration explicite est laissée par Foucault à un prochain livre.

\section{Partie 3}

3. Il y a plusieurs des thèmes développés par $\mathrm{M}$. Foucault, dont nous n'avons rien dit jusqu'ici et qui méritent notre attention. Nous occuperons cette section à en rendre compte.

3.1 Nous avons déjà dit que nous ne pensions pas que M. Foucault fournît une démonstration soutenue de l'intrication des divers réseaux $\mathrm{du}$ savoir et du pouvoir, dont l'affirmation fonctionne dans le texte davantage à titre de leitmotiv qu'à titre de notion thématique. Il s'en faut de beaucoup cependant pour qu'on puisse lui reprocher d'affirmer de façon gratuite l'existence d'une telle collusion. Il suffit pour le montrer de rappeler les grands traits de cette esquisse de la généalogie des sciences humaines qu'il nous soumet. Les positions qu'il développe se rapprochent de certaines des propositions de la psychologie criminologique, en même temps qu'elles en soulèvent l'ambiguiité. L'esquisse présentée par M. Foucault est, en gros, la suivante. On peut, se plaçant à un très haut niveau d'abstraction, contraster les sciences humaines avec les sciences de la nature en arguant que les secondes sont conformes au précepte aristotélicien selon lequel il n'y a de science que du général alors que les sciences humaines, et de façon particulièrement évidente la psychologie clinique, essaient au contraire d'élaborer un savoir de l'individu. On peut dès lors penser que l'émergence de la notion d'individualité conditionne la constitution des sciences humaines. La notion d'individuation qui existait avant la période faisant l'objet des analyses de M. Foucault était en quelque sorte aspirée par le haut et coïncidait avec un processus social d'héroïsation - seuls les dieux, les héros ou les princes étaient reconnus dans leur singularité - qui donnait davantage lieu à une mythologie qu'à une science. Pour 
qu'un savoir émerge, qui ne fût pas soupçonné d'être une profanation de personnages sacralisés, il a fallu inverser vers le bas le processus d'individuation. C'est ce dont les sciences humaines sont redevables au complexe carcéro-judiciaire.

Il faut d'abord remarquer sur ce sujet que la déviance ou l'anormalité pactisent au regard de l'intuition naïve avec l'individualisation. Ce qui s'écarte de la norme est d'emblée préparé à être investi des prédicats de la singularité. C'est en fait la notion de récidive qui contribuera à opérer cette métamorphose des propriétés d'un comportement en des attributs d'une personnalité, dès lors posée dans son individualité (déviante). L'acte ne possédant pas en lui-même la raison de sa répétition, on lui suscite un noyau étiologique qui est ensuite réifié sous la forme d'une personnalité ou d'une individualité délinquantes. Cette genèse de la notion d'individualité allait avoir des conséquences capitales dans la détermination des objectifs du système pénal. Il n'y a en effet aucune rémission à l'acte criminel comme tel. Son trait définitoire est d'avoir été accompli et de ne pouvoir en conséquence que susciter une punition. Une personnalité délinquante peut au contraire faire l'objet d'un redressement et d'une transformation: d'où l'investissement du système carcéral dans les projets de réhabilitation.

On ne peut, je crois, manquer d'être frappé par les analogies entre le processus de genèse de la notion de personnalité délinquante, telle que le décrit $M$. Foucault, et la projection sur un individu d'une identité négative qui est accomplie par divers processus sociaux ou familiaux de stigmatisation. La psychologie et la sociologie criminelles ont maintes fois dénoncé les effets désastreux sur un individu de ces processus de stigmatisation. Ces dénonciations, à la lumière des analyses de Michel Foucault, ne laissent pas d'apparaitre ambiguës. La psychologie s'est, pour une, investie de façon massive dans la réalisation de projets carcéraux de réhabilitation. Cet investissement semblerait indiquer que le concept de personnalité délinquante y demeure encore prégnant d'une valeur de vérité certaine. On pourrait en induire que les mises en garde de la psychologie et de la sociologie criminelles ne constituent que des avertissements relativement bénins qui s'adressent avant tout à une pratique empirique et naïve - familiale - de la stigmatisation mais qui se gardent bien d'entamer la pierre angulaire du processus de stigmatisation, c'est-à-dire la croyance en l'existence de personnalités délinquantes. Toute la question est évidemment de savoir si c'est la naïveté populaire ou la complicité prestigieuse du savoir scientifique qui est la 
caution la plus opérante du système carcéral. Dans la mesure où ce système se définit comme une technique de redressement qui, comme toutes les techniques, tire sa légitimité de ce qu'il est informé par un savoir, on peut penser que c'est le pacte qu'il a passé avec les réseaux de la production scientifique qui est le plus essentiel à son maintien. C'est d'ailleurs le prestige qui est conféré à certaines institutions carcérales, à la suite de l'existence de cette alliance avec le savoir, qui entretient pour une large part la ferveur populaire.

3.2 Il est une autre des transformations de la pénalité, dont Michel Foucault nous décrit la genèse et qui vient croiser, pour les confirmer, certaines des positions défendues par Sutherland ${ }^{3}$ au sujet de la criminalité des cols blancs. Le début du XIx ${ }^{\mathrm{e}}$ siècle, affirme Foucault (p. 89 et suiv.), a été témoin d'une restructuration de l'économie des illégalismes qui a tiré une ligne de partage entre l'illégalisme des biens et l'illégalisme des droits. Dans ce partage, la bourgeoisie s'est attribué l'illégalisme des droits - fraude, évasion fiscale et opérations commerciales irrégulières - tout en laissant aux classes populaires l'illégalisme des biens. Il faut à cet égard préciser que la dichotomie qui est établie entre ces deux types d'illégalités est profondément discriminatoire et désigne plutôt deux modes de poursuite judiciaire que les objets véritables d'opérations illégales dont la différence viendrait d'ailleurs que de la simple échelle de leurs bénéfices. L'illégalisme des droits porte autant, et même davantage puisque ses profits sont sans commune mesure avec ceux de l'illégalisme des biens, sur les biens - l'argent qui s'accumule sous forme de capital - que cette forme d'illégalisme qui est explicitement désigné comme illégalisme des biens. Seul ce dernier est cependant stigmatisé comme vol et ressortit à la branche criminelle du droit alors que l'illégalisme des droits a été codifié par le droit civil ou fait l'objet de juridictions d'exception. C'est dans cette assignation au droit criminel de la partie la plus dramatisée, bien que la moins lucrative, des illégalismes que se manifeste la mainmise de ceux qui avaient le pouvoir d'édicter les lois - à toutes fins pratiques, la classe bourgeoise - sur l'illégalisme des droits, placé hors de la portée des châtiments corporels. De la même façon que la guerre n'est, d'après Clausewitz, que la poursuite à l'aide d'autres moyens des relations politiques entre

3. Voir l'article d'André Normandeau (1970), Les déviations en affaire et les crimes en col blanc. In D. Szabo (Ed.), Déviance et criminalité (32-51). Paris: Armand Colin. 
les États, on peut dire que la procédure judiciaire civile, qui dans un nombre considérable de cas donne lieu à des arrangements hors des tribunaux, n'est que la poursuite des relations commerciales entre négociants rivaux - le plus gros d'entre eux étant l'État - bien que par d'autres moyens.

3.3 Michel Foucault fixe la date de la formation du système carcéral au 22 janvier 1840 (p. 300). Cette date, qui est celle de l'ouverture de la colonie pénitentiaire pour jeunes délinquants de Mettray, prend la valeur d'un symbole. Ce symbole est celui du débordement de la prison sur un ensemble d'institutions - écoles de réforme, hospices pour vieillards, asiles d'aliénés - qui se transforment de façon croissante en des maisons disciplinaires et finissent par constituer un réseau carcéral faisant système. Le système carcéral compose ensemble quatre éléments (p. 276): 1) un supplément disciplinaire, dont il réclame la gestion autonome, et qui est à l'origine de son débordement sur d'autres institutions qui affirmeront reprendre à leur compte le projet de réformer les âmes en disciplinant les corps; 2) la production d'un nouveau champ d'objectivité, les corps à soumettre et leurs corrélats spirituels, les personnalités délinquantes, qui donnera lieu à des recherches adonnées à produire un nouveau savoir; 3) une fonctionnalité inversée: le système carcéral produit la délinquance plutôt qu'il ne la réduit; 4) une mythologie de la réforme qui est le masque qu'y prend l'utopie. Le système carcéral, dont les défauts ont été aperçus dès son instauration, est en effet depuis ce temps soumis à un processus de révision perpétuel, qui n'arrive qu'à reproduire le semblable en espérant toujours faire une différence.

Telles sont les propriétés que l'on pourrait qualifier de structurales du système pénal, pour les contraster avec une caractéristique de ce système, qui se manifeste si l'on articule selon des séquences temporelles les différents termes qui le constituent, au lieu de les considérer dans leur simultanéité. Le propre des diverses maisons qui appartiennent au système carcéral est que, aboutées les unes aux autres, elles peuvent être mises en correspondance avec les divers âges d'une vie humaine, qu'elles croisent alors sur toute sa longueur, permettant l'initiation et la poursuite de carrières carcérales qui commencent dans les maisons pour enfants trouvés et qui se terminent à l'hospice, quand elles n'ont pas été interrompues de façon définitive par la caserne. La crèche, le foyer nourricier, l'école de réforme, les colonies de la Cour du bien-être social, le pénitencier, les officines de l'assistance sociale et celles de 
l'assurance-chômage, l'hôpital et finalement l'hospice: déploiement sans grande surprise d'un tragique humilié où les protagonistes n'ont même pas la ressource de se crever les yeux pour cesser de voir ce qu'on fait de leur pauvre vie, puisqu'ils n'ont jamais été fautifs. Si le carcéral ne remplit pas encore tout notre espace, il peut du moins revendiquer de requérir tout notre temps.

3.4 Nous aimerions dans cette dernière section de la troisième partie de notre exposé souligner l'utilisation prospective que l'on peut faire des recherches de M. Foucault. Revenons d'abord brièvement sur des notions qui ont déjà été présentées. On peut, avons-nous dit, distinguer trois états dans l'évolution de la pénalité en Occident. Premier état, une pénalité des corps suppliciés. Second état, le projet de créer un système pénal qui serait co-extensif à toute la sphère de l'illégalisme des biens. Ce projet, nous l'avons aussi vu, a été contraint de concevoir sa réalisation sous la forme d'une sémio-technique qui projetait de lier dans la conscience de la communauté l'idée de la certitude du châtiment avec celle du crime. Mais si l'imposition de ce modèle a été court-circuitée par la mise en place d'un troisième modèle - le carcéral - qui dévia à nouveau sur les corps la visée des systèmes pénaux, il faut néanmoins souligner avec force que le modèle carcéral a repris à son compte l'une des innovations les plus essentielles des premiers réformateurs de la pénalité des supplices. Cette nouveauté consistait dans la découverte de l'exigence d'amplifier considérablement la prise du système pénal sur une communauté déterminée, en ayant recours à une généralisation de la surveillance. La reprise de cette exigence par la pénalité carcérale est attestée de plusieurs manières par Michel Foucault. D'abord par le relais qu'il constate, dans sa description de la montée des disciplines, entre une discipline-blocus qui est instaurée par un état d'exception (la peste) et une discipline-mécanisme dont le terme réside précisément dans l'organisation d'une surveillance généralisée (p. 211). Ensuite lorsqu'il subsume sous le terme de panoptisme la somme des finalités qui présidèrent à la mise en place du carcéral et de l'idéologie qui lui servait de justification.

D'où la présence au sein du système carcéral d'une tension, qui au regard de ses finalités, a déterminé son échec profond. Il était en effet impossible aux réformateurs du $\mathrm{XIX}^{\mathrm{e}}$ siècle, de quelque tendance qu'ils fussent, de concilier avec la technologie dont disposait l'époque les impératifs d'instaurer une surveillance générale et de lui donner pour 
objet les corps. Si l'on doit penser que la réforme d'une pénalité des supplices a achoppé parce qu'elle a donné lieu à un système - le système carcéral - qui a perverti l'une de ses exigences fondamentales en châtiant à nouveau les corps, il faut aussi conclure que le système carcéral constitue lui-même un vaste échec puisqu'il achoppe à donner une forme satisfaisante à la seconde et à la plus essentielle des exigences qui définissait les principes de la nouvelle pénalité, à savoir l'instauration d'une surveillance généralisée qui réussirait à établir un quadrillage exhaustif du corps social. Le panoptisme n'a été réalisé qu'à l'intérieur de ces environnements privilégiés que sont les prisons. Et encore. Il est sûr qu'à entendre ce terme au sens strict, il n'est pas une seule de nos prisons qui soit une institution véritablement panoptique, c'est-à-dire où l'on puisse observer tout ce qui s'y passe.

L'objet de la thèse historique, nous l'avons souvent rappelé, est de rendre compte pourquoi l'intention de substituer à une pratique des supplices une pénalité du signe s'adressant à l'esprit, a subi cette torsion qui a donné naissance au système carcéral. On peut s'étonner que M. Foucault n'ait pas eu recours pour expliquer, ne serait-ce qu'en partie, cette perversion des intentions premières à une hypothèse qui semble relativement obvie. Selon cette hypothèse, le carcéral ne constituerait qu'une étape préparatoire imparfaite dans la réalisation de l'objectif essentiel de généraliser la surveillance. L'intermède carcéral aurait été rendu nécessaire par suite du manque de moyens techniques pour réaliser cette généralisation de la surveillance qui constituait l'alternative initiale à une pénalité des supplices. Ne pouvant s'appuyer sur les ressources contemporaines de la propagande, le projet de contraindre les âmes a dû, au XIx $x^{e}$ siècle, ajourner sa réalisation systématique au profit d'une phase expérimentale où s'élaborerait dans des prisons-laboratoires la technologie permettant d'asservir les âmes à travers une domestication des corps.

Or nous possédons aujourd'hui les moyens de généraliser la surveillance, quelle que soit sa variante, et nous nous y employons. Nous investissons d'abord les âmes et les cerveaux par le moyen de la propagande et des techniques de rupture des volontés. Nous avons en outre, et de façon croissante, le pouvoir de garder les corps à vue. Il est intéressant à cet égard de rapprocher le chapitre que Michel Foucault consacre au panoptisme à la description cauchemardesque de la société future qui est proposée par George Orwell dans son œuvre célèbre intitulée 1984: les murs des villes y sont couverts d'affiches où il est 
inscrit «Big Brother is looking at you» en dessous de la reproduction d'un visage aux yeux démesurés. À n'en pas douter, le quadrillage de l'espace social que souhaitaient les auteurs de Codes pénaux du XIX siècle sera bientôt réalisé: il ne reste que de savoir s'il s'achèvera sous la forme d'un panoptisme ou d'un panacoustisme, le ruban magnétique disputant actuellement à la technique photographique l'honneur d'être l'opérateur privilégié de la surveillance. L'un des symptômes de l'engagement de nos sociétés dans une généralisation de tous les mécanismes de surveillance tient dans ce fait que les plus libérales d'entre elles affirment voir dans l'intensification de la surveillance l'alternative véritable au système carcéral. Le cas de la Hollande est à cet égard significatif $^{4}$. On pourrait dès lors se représenter l'établissement d'une surveillance généralisée comme un processus en voie de finalisation et qui s'est instauré en deux étapes, après avoir dû procéder à deux déplacements. Ayant d'abord à demi achoppé à se substituer à une pénalité des châtiments corporels, le projet de surveiller a dû attendre de posséder les moyens techniques de sa réalisation pour ambitionner à nouveau de déplacer l'avatar moderne d'une pénalité des corps, la prison. Si cependant on voit, comme, croyons-nous, il faut le faire, dans le système carcéral la mise en place d'un programme de surveillance absolue, qui s'articule sur la notion de panoptisme, on doit alors conclure que le remplacement des maisons de détention par une intensification de l'inspection policière, au lieu de constituer une alternative au carcéral, ne représente en définitive que la réalisation enfin adéquate de son espoir le plus démesuré. L'instauration de la surveillance, loin de constituer l'expulsion de la prison hors de nos cités, signifie au contraire l'intégration de tout l'espace social, transformé en un gigantesque Panopticon, au système carcéral.

\section{Partie 4}

4. Nous allons maintenant procéder à un examen critique de ce qui nous paraît constituer certaines difficultés de méthode dans Surveiller et punir. Qu'il soit cependant entendu qu'il n'est aucune de ces difficultés dont l'ampleur soit comparable à l'estime que l'on doit porter au travail de Michel Foucault.

4. Voir par ex. L. C. H. Hu1sman (1974), Criminal Justice in the Netherland. Delta, p. $7-19$, p. 10. 
4.1 Surveiller et punir constitue à au moins un égard une innovation dans l'œuvre de Foucault. Il s'était jusque-là davantage occupé de retracer la genèse de concepts (la folie), de méthodes (le regard médical) ou celle de différentes disciplines scientifiques. Le commun dénominateur de ces objets est qu'ils appartiennent au domaine des productions de l'esprit, dont la manifestation historique habituelle est constituée par un ensemble de textes. Il est dès lors normal et attendu que les données de base auxquelles doit avoir recours celui qui se livre à une genèse des notions ou à une archéologie des savoirs aient la forme de textes. Or il doit en aller autrement lorsque l'on ambitionne de faire l'histoire de processus sociaux qui se sont objectivés sous une autre forme que sous celle de la production de textes et qui ont plutôt donné naissance à ce fait massivement empirique qu'est le système carcéral. En d'autres termes, relate-t-on la naissance des choses de la même manière dont on rend compte de celle des mots? Assurément, il n'est pas de discipline historique dont les données fondamentales ne soient constituées par des textes. Notre accès au passé doit nécessairement être médiatisé par ses traces textuelles, qui seules constituent le réceptacle de ce qui n'est plus. Il y aurait cependant lieu à cet égard de distinguer entre une matière textuelle constituant les "hard datas» de l'histoire et que l'on pourrait désigner comme des documents et un autre type de textes constituant plutôt des «soft datas» et auquel on continuerait de réserver l'appellation de textes; seuls les documents consignent les faits alors que les textes ne gardent que la mémoire des idéologies. Que l'on compare à cet égard la matière textuelle qui sert de base aux travaux d'historiens comme Roland Mousnier ${ }^{5}$ ou comme Pierre Chaunu ${ }^{6}$ à celle sur laquelle se fonde Michel Foucault. Les premiers privilégient nettement le document - livres de comptes, registres d'État, statistiques - alors qu'il nous semble que les ouvrages auxquels se réfère Michel Foucault constituent davantage des textes rédigés tantôt par des juristes, des idéologues ou des journalistes. Il faudrait cependant, pour rendre justice à Foucault, faire la statistique des textes qu'il cite dans les notes au bas des pages. Le privilège que nous pensons qu'il accorde à des soft datas tient à des impressions de lecture et surtout à l'absence constatée de renvois à des documents faisant état de données quantitatives. Si cette primauté du texte sur le document dans le livre de Foucault est véritable, la question

5. Roland Mousnier, La vénalité des offices sous Henri IV et Louis XIII, Rouen, 1945.

6. Pierre Chaunu, Séville et l'Atlantique (1504-1656), Paris, $1^{\text {re }}$ partie, 7 tomes, 8 volumes, 1955-1957 et $2^{\mathrm{e}}$ partie, 4 volumes, 1959-1960. 
qui se pose dès lors est la suivante: faire l'histoire de l'arrière plan intentionnel ou idéologique sur lequel s'est profilée la naissance de la prison équivaut-il à rendre compte de la genèse de la prison elle-même? Quoi qu'il en soit de la réponse que l'on apportera à cette question, une chose est certaine: on doit considérer que la base empirique sur laquelle se fonde ce que nous avons appelé la thèse socio-judiciaire de Foucault est nettement insuffisante.

4.2 On doit noter en second lieu une considérable asymétrie entre le degré de généralité des données présentées par Michel Foucault et le type de thèse qu'il nous propose. Ceci est particulièrement visible dans le cas de la thèse épistémologique. Le cadre spatio-temporel de la recherche de Michel Foucault semble, au premier abord, déterminé avec précision. Il s'agit d'une histoire de la naissance de la prison dans le seul système français (p. 35, note 1); cette histoire s'étend en outre du $\mathrm{XVI}^{\mathrm{e}}$ au XIX ${ }^{\mathrm{e}}$ siècle. On s'explique dès lors assez mal que Foucault s'appuie sur des recherches dont le cadre est ainsi déterminé pour tirer des conclusions qui concernent la formation des sciences humaines, la naissance des idéologies qui s'élaborent dans l'horizon de l'humanisme occidental et la fonction contemporaine de l'échec du système carcéral (la question qui donne lieu à la thèse socio-judiciaire est formulée au présent. Voir p. 277). Bien évidemment, Michel Faucault ne croit pas que les sciences humaines, pas plus que l'humanisme moderne, sont nés en France. On doit donc conclure qu'il attribue au développement de la pénologie en France une valeur paradigmatique, qui serait confirmée par l'histoire du développement des systèmes pénaux dans d'autres pays. Rien ne nous assure cependant de cette confirmation.

4.3 Ce débordement du cadre de la recherche par les thèses qu'on lui permet d'autoriser a pour conséquence, par choc en retour, d'introduire d'importantes marges d'imprécision quant aux limites de validité effective des propositions du livre et quant à la manière de la vérifier. Cette imprécision est particulièrement sensible dans la dernière partie de l'ouvrage, où l'argumentation développée hésite constamment entre la thèse historique et la description sociologique du système carcéral actuel et sa fonction dans la société contemporaine. Or tout discours qui prend pour objet la problématique de l'enfermement au $\mathrm{xx}^{\mathrm{e}}$ siècle doit selon nous prendre position à l'égard de la nature du phénomène concentrationnaire, qui semble être l'apport propre de notre siècle au développement de la 
pénalité. Quel est le rapport du système concentrationnaire avec le système carcéral? En est-il l'excroissance monstrueuse ou constitue-t-il quelque chose de fondamentalement différent? L'ambiguiité relative des bornes de la recherche de Michel Foucault ne nous permet malheureusement pas de savoir si ces questions sont pertinentes pour elle.

4.4 Si maintenant nous quittons le terrain des données utilisées par Foucault pour nous interroger sur le type de raisonnement qu'il utilise, nous ferons la remarque suivante. La procédure la plus habituellement suivie par M. Foucault est de conclure d'une homologie ou de ressemblances fonctionnelles - repérées avant tout par le moyen de l'analyse de textes énonçant des intentions et déterminant des objectifs - entre des processus ou des systèmes à une filiation génétique effective dans l'ordre concret de l'histoire. Il est très difficile de déterminer si les techniques disciplinaires et les pratiques d'incarcération sont tout simplement juxtaposées dans Surveiller et punir ou si Michel Foucault nous fournit pour expliquer la colonisation des secondes par les premières un autre opérateur que leurs seules analogies de fonction. Le Panopticon dont Bentham nous fournit l'esquisse - reproduite dans les planches du volume de Foucault - ressemble comme un frère à nos prisons. Doit-on conclure à l'influence effective de Bentham sur la formation du système carcéral? Léonard de Vinci a bien dessiné avec une grande précision des avions et même des hélicoptères; sa part dans le développement de l'aéronautique peut cependant être considérée comme négligeable.

4.5 Dernière remarque, enfin, pour souligner le paradoxe auquel nous conduit la thèse de Foucault sur la fonction qui doit être attribuée à l'échec du système carcéral. Cette fonction est de permettre un contrôle de la délinquance et une gestion efficace des illégalismes. Or c'est précisément ce contrôle de cette gestion qui, eu égard aux finalités que poursuit un système pénal, constituent les critères les plus habituels pour évaluer son succès. Il se révèle donc, de façon peut-être un peu paradoxale, que c'est l'échec même du système carcéral qui constitue la plus sûre garantie de sa réussite. Un pouvoir politique qui favoriserait le pragmatisme sur la moralité en conclurait que ce système doit être maintenu et son échec entretenu. Le plus rentable des systèmes est celui qui réussit à produire son énergie à même son déchet. Tel est le système carcéral; cette conclusion a de quoi décourager une stratégie de changement dans le domaine de la pénalité. 


\section{Partie 5}

5. La section précédente peut servir de conclusion à notre brève exégèse du livre de Michel Foucault. Il ne nous reste plus dès lors qu'a répondre à une question: qu'est-ce que les recherches criminologiques peuvent retenir du livre de Michel Foucault, à part bien entendu son grand intérêt documentaire? Il serait présomptueux de notre part de vouloir répondre de façon exhaustive à cette question. Aussi allons-nous nous contenter de ne livrer que quelques réflexions où nous utiliserons l'expression «la criminologie» davantage pour référer à des enseignements qui nous ont été présentés que pour désigner la masse des recherches criminologiques, dont nous ne possédons pas encore la maîtrise.

5.1 Il nous semble d'abord que Michel Foucault nous convie à approfondir la signification véritable de la collusion qui s'est établie entre les réseaux du savoir et le pouvoir politique. Celle-ci est habituellement conçue, de façon relativement superficielle, d'abord comme la complaisance du savoir à laisser le pouvoir politique exploiter les résultats auxquels elle parvient à la suite de recherches désintéressées et ensuite comme un acquiescement occasionnel à une directive politique qui l'inviterait à porter provisoirement, à l'intérieur du champ de phénomènes qu'elle s'est assigné en ne doutant pas de sa réalité, son attention sur certains objets plutôt que sur certains autres. Le livre de Michel Foucault donne à entendre que les conséquences de l'édification d'un complexe scientifico-politique sont beaucoup plus considérables que ne le suppose cette représentation naïve. Loin de se contenter d'infléchir le regard à l'intérieur d'un champ d'objets pré-existants que le savoir délimite de façon autonome, le politique peut être avant tout redevable de la constitution même ex potestate, de tout un nouveau champ d'objets, dont les bornes sont fixées arbitrairement par les requêtes d'une conjoncture et dont la réalité doit par conséquent être soupçonnée. L'orientation prise par les recherches criminologiques porte témoignage de cette opération du politique dans le domaine épistémique. C'est en effet un des lieux communs de la philosophie du droit que le crime désigne une relation entre un comportement et la norme qu'il transgresse. D'où il aurait dû suivre que les études criminologiques portassent à la fois sur les comportements réputés criminels et sur les divers systèmes définitoires - judiciaires, policiers ou autres - constituant le corrélat nécessaire de ce qui est posé comme crime. Au lieu de cela, la 
criminologie, quand elle ne s'employait pas à raffiner les techniques policières, s'est pour une part très considérable de son activité investie dans une plate psycho-socio-genèse qui ruse d'imagination pour éviter de rapporter le crime à ce qui le constitue en lui-même, à savoir le système pénal. Le livre de Michel Foucault est un rappel péremptoire de la nécessité de mener conjointement les études sur la criminalité avec des recherches portant sur le système judiciaire et ses diverses ramifications. Il serait même souhaitable que ce dernier prenne provisoirement la première place dans nos préoccupations afin de rétablir un équilibre depuis longtemps compromis.

5.2 Si la puissance politique peut provoquer des déblocages épistémologiques qui aboutissent à la constitution de tout un champ de phénomènes, il devient manifeste que les discours qui prennent pour objet ces nouvelles atlantides que le pouvoir fait brusquement émerger de la marée de l'histoire devront procéder à un re-examen constant et sévère de la valeur de vérité de leurs principaux concepts et notions fondatrices. Si le concept de délinquance, pour prendre un exemple, n'est que le fantôme étiologique qu'une volonté de contrôle et de redressement s'est donné pour se légitimer, il devient évident que des oppositions comme celles que l'on fait couramment entre la criminalité et la délinquance sont dépourvues de pertinence et de fondement. On pourrait citer de la même façon plusieurs des notions que véhicule la criminologie.

5.3 Il est enfin deux points de nature méthodologique sur lesquels nous aimerions dire quelques mots en terminant. La criminologie, nous semble-t-il, est génétique là où elle devrait être structurale et elle demeure naturaliste et fascinée par la description des substances là où elle devrait écrire l'histoire des processus. Expliquons-nous. Lorsqu'elle aborde ses objets selon une coupe synchronique - description de la criminalité à une époque donnée, considérée en elle-même - la criminologie, curieusement, temporalise ses concepts et nous fournit sous l'espèce des diverses psycho et socio genèses des histoires de cas. Dit autrement, elle privilégie la chronique des vies individuelles là où elle devrait décrire des structures, puisqu'il est acquis que la criminalité constitue une relation dont les termes se présupposent réciproquement, sont en conséquence simultanés et doivent donc faire l'objet d'analyses conjointes. D'autre part, parce qu'il n'est pas d'emblée refléchi comme une relation, le crime est posé comme une substance, dont la nature, 
comme celle de toutes les substances, est en son fond essentiel inaltérable; il en suit que les recherches criminologiques ont donné lieu à un nombre assez restreint de recherches de nature historique, si nous les comparons à la masse des études synchroniques. Il y aurait selon nous un profit immédiat pour la criminologie à substituer la notion de criminalisation au concept de crime. Désignant un processus plutôt qu'une entité reposant en elle-même, cette notion pourrait entraîner une multiplication des recherches dont nous déplorons, peut-être à tort, le nombre trop restreint. Devant en effet rechercher l'agent du processus de criminalisation - le judiciaire - on abordera dès lors de façon obligée l'étude de la criminalité de façon structurale. Reconnaissant d'autre part que ce processus se déploie dans le temps, on en viendra à produire des études historiques dont le livre de Michel Foucault nous donne un exemple si stimulant.

ABSTRACT - In this paper, we will start by presenting, in schematic form, the principal theses discussed in Michel Foucault's book. Then, we will devote the second part to a deeper understanding of the principal parts of the argumentation he develops and we will see how some of the themes he develops are of a more immediate interest to the criminologist, either because they converge with research in criminology or because they constitute a critique of it. Then, we will raise certain points that concern the method used by Foucault. This part of our argument will be more critical. Finally, we will conclude with a recall of Foucault's conclusions on the birth of criminology. We will then attempt to shed light on what criminological studies should retain of Michel Foucault's book, furthermore about what he has taught us, from a documentary point of view, of the birth of the prison.

KEYWORDS - Michel Foucault, prison system, sentencing, panopticism, hard data, soft data.

RESUMEN - En la primera parte de este artículo presentamos, de forma esquemática, las principales tesis defendidas en el libro de Michel Foucault. En la segunda parte, examinamos los principales puntos de la argumentación que desarrolla y mostramos cómo algunos de los temas del libro resultan de interés para la criminología, ya sea porque convergen con la investigación en criminología o porque constituyen una crítica a ésta. A continuación, analizamos algunos aspectos de la metodología empleada por Foucault -esta sección resulta más crítica. Para terminar, recordamos las conclusiones de Foucault sobre el nacimiento de la criminología y subrayamos los elementos que los estudios criminológicos deben retener del libro de Michel Foucault, además de aquellos, de índole documental, relacionados con el nacimiento de la criminología.

PALABRAS CLAVE - Michel Foucault, prisión, sistema penitenciario, penalidad, panoptismo, hard datas, soft datas. 